\title{
TRAITEMENT D'URGENCE DES RADIOCONTAMINATIONS RESPIRATOIRES : GÉNÉRATEURS AUTONOMES D’AÉROSOLS DE DTPA.
}

\author{
R. DUCOUSSO, E. GIRERD, C. PASQUIER (*)
}

(Manuscrit reçu le 30 septembre 1974)

\begin{abstract}
RESUME
Des études préalables ont montré la nécessité d'inhaler un aérosol de D.T.P.A. sur simple présomption de contamination respiratoire par des radionucléides tels que le plutonium et les transplutoniens, l'yttrium et les terres rares, ce, le plus tôt possible après l'accident. Pour que ce traitement d'urgence puisse être mis en œuvre, les personnels exposés doivent disposer d'un générateur d'aérosol individuel et portatif.

Dans ce travail, nous avons essayé deux appareils susceptibles de répondre aux critères imposés par le problème à résoudre. L'un d'entre eux, le turbo-inhalateur SPINHALER semble devoir convenir de façon très satisfaisante.
\end{abstract}

\begin{abstract}
Previous experiments have demonstrated that D.T.P.A. aerosols should be administered by inhalation as soon as possible following a suspected accidental inhalation of plutonium, transplutonic elements, yttrium or rare earths. This emergency treatment requires that each exposed individual should have his own portable aerosol generator.

Two generators were tried; the SPINHALER turbo-inhalator seemed to answer the conditions required satisfactorily.
\end{abstract}

\section{INTRODUCTION}

L'administration du sel monocalcique et trisodique de l'acide diéthylènetriamine-pentacétique (D.T.P.A.) est indiquée dans tous les cas de contamination interne par des radionucléides de valence égale ou supérieure à trois [1], sauf dans le cas du neptunium [2] et de l'uranium [3]. Son action

(*) Centre de Recherches du Service de Santé des Armées et Commissariat à l'Energie Atomique (D.Pr.) (contrat 52-02), BP n ${ }^{\circ}$ 6, 92260 Fontenay-aux-Roses (France).

RADIOPROTECTION, VOL. 9 - $\mathrm{N}^{\circ} 3$ 
chélatrice ne s'exerce que vis-à-vis des ions. Par conséquent, son efficacité thérapeutique est liée à l'état physico-chimique du radionucléide en cause lors de la contamination. Ainsi, l'action thérapeutique du D.T.P.A. est pratiquement nulle vis-à-vis des formes chimiquement insolubles telles que les métaux ou les oxydes calcinés ; de plus, cette action thérapeutique diminue très rapidement avec le temps vis-à-vis des formes chimiquement solubles (nitrates, chlorures) du fait de l'hydrolyse et de la polymérisation de celles-ci. Nous avons montré par ailleurs l'importance de cette perte d'efficacité en fonction du temps [4]. De ce fait, lors d'un incident, la simple présomption d'une contamination interne doit déclencher la mise en œuvre d'un traitement d'urgence avant même de pratiquer le diagnostic de certitude [5].

Dans le cas de contamination respiratoire par radionucléides relevant d'une thérapeutique par le D.T.P.A., l'efficacité de l'administration locale du chélatant sous forme d'aérosol a été notée par de nombreux auteurs $[1,6,7]$. Nous avons nous-mêmes étudié quantitativement les différences d'efficacité selon la voie d'administration [4] et tenté d'en donner une explication [8].

La combinaison des deux concepts, d'urgence thérapeutique d'une part, et d'administration locale d'autre part, nous a conduits à entreprendre la mise au point d'un procédé d'inhalation du D.T.P.A., individuel et portatif, que chaque personnel soumis au risque de contamination respiratoire par yttrium, lanthanides, plutonium et transplutoniens pourrait avoir à sa disposition, au même titre qu'un appareil respiratoire filtrant.

\section{CRITERES D'UN DISPOSITIF D'INHALATION INDIVIDUEL ET PORTATIF}

Les caractéristiques d'un dispositif d'inhalation individuel et portatif sont forcément différentes de celles d'un générateur d'aérosols médicamenteux classique, ne serait-ce que par le fait qu'il faut se débarrasser du gaz moteur conventionnel qu'est l'air comprimé, obtenu à partir d'une bouteille ou d'un compresseur. On peut retenir les critères suivants :

1. Innocuité : il ne s'agit pas là de celle du principe actif qui est en principe admise, mais de celle des adjuvants (gaz propulseur, stabilisateur d'émulsion, etc.). naire.

2. Efficacité : le principe actif doit pouvoir atteindre l'alvéole pulmo-

3. Taille : l'appareil doit pouvoir mériter l'appellation « de poche».

4. Simplicité d'emploi : l'utilisation doit être à la portée de tous et ne poser aucun problème, même si le sujet a perdu une partie de ses moyens après un accident.

5. Sûreté de fonctionnement: le risque de non fonctionnement ou d'arrêt de fonctionnement en cours d'utilisation doit être nul.

6. Conservation des propriétés du principe actif : elle doit être assurée 
en particulier du point de vue de l'efficacité thérapeutique, de la stérilité et de la granulométrie.

7. Prix de revient : il doit être faible, puisque le dispositif est individuel, donc destiné à être largement distribué.

Deux appareils semblaient pouvoir répondre à ces charges :

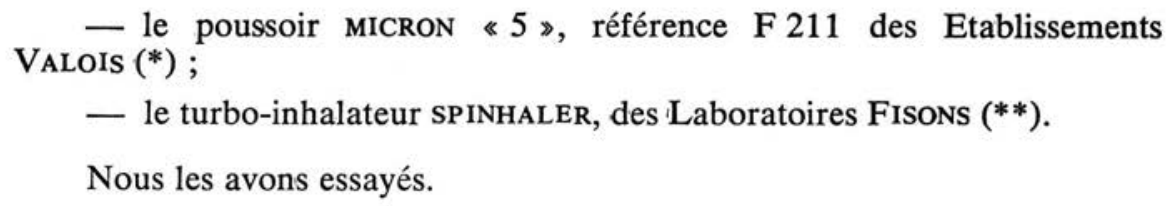

\section{MATERIELS ET METHODES}

$1^{\circ}$ D.T.P.A.

Le D.T.P.A. a été utilisé sous sa forme monocalcique et trisodique, en poudre micronisée. En effet, le SPINHALER est un générateur d'aérosols solides et le poussoir MICRON « 5 » doit utiliser le fréon pour propulser particules ou gouttelettes jusqu'à l'alvéole pulmonaire ; or, la solution aqueuse de D.T.P.A. n'est pas utilisable parce que non miscible au fréon et le D.T.P.A. n'est pas assez soluble dans l'alcool pour que ce solvant puisse être employé.

La granulométrie de la poudre de D.T.P.A. micronisée est telle que $80 \%$ des particules a moins de $2 \mu \mathrm{m}$ de diamètre.

La figure 1 montre le diagramme de fréquence en pourcent de la répartition en dimension des particules. Les mesures ont été faites au microscope optique $(G=800)$ sur 700 éléments environ.

\section{$2^{\circ}$ RADIONUClÉIDES}

Nous avons utilisé le lanthane 140 en raison de sa faible radiotoxicité et parce que, chef de file des lanthanides, il peut servir de modèle pour ceux-ci, ainsi que pour pratiquement tous les éléments hydrolysables, plutonium et transplutoniens compris.

Dans nos conditions, les principaux paramètres influant sur l'efficacité du D.T.P.A. administré en aérosol sont la charge pulmonaire initiale en lanthane [9] et le délai d'administration du chélatant [4]. Nous avons fixé pour toutes les expériences la charge pulmonaire initiale à $10 \mu \mathrm{g}$ et le délai d'administration du D.T.P.A. à $30 \mathrm{mn}$ après la contamination.

(*) Etablissements VALOIS : avenue de l'Europe, 78160 Marly-le-Roi.

(**) Laboratoires FISONS : 24, rue des Ursulines, 93202 Saint-Denis.

voL. $9-\mathrm{N}^{\circ} 3$ 


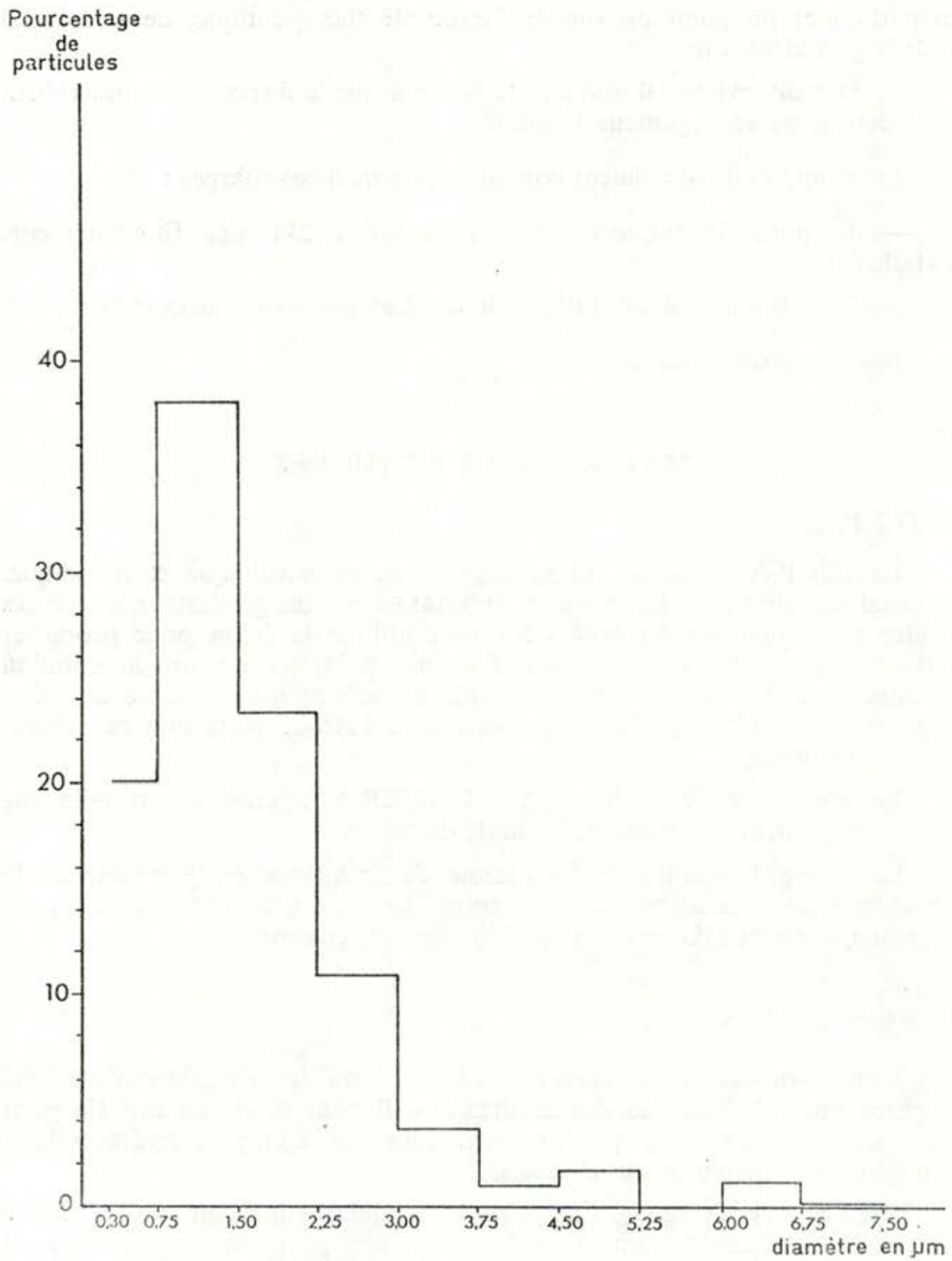

Fig. 1. - Diagramme de fréquence de la répartition en dimension des particules de D.T.P.A.

\section{$3^{\circ}$ ANIMAL}

Pour les essais du MICRON « 5 » nous avons utilisé deux singes Babouins d'environ $3 \mathrm{~kg}$. 
Le SPINHALER, du fait de son principe de fonctionnement, ne se prête pas à l'utilisation dans le circuit d'un appareil de respiration assistée. Nous l'avons essayé sur un volontaire humain.

\section{$4^{\circ}$ Technique D'inhalation du Radionucléide}

La technique d'inhalation du lanthane par l'animal a été précédemment décrite [10]. Elle favorise le dépôt alvéolaire aux dépens du dépôt bronchique et permet de standardiser les paramètres respiratoires.

La technique utilisée chez l'Homme est celle de Gongora et coll. [11].

\section{$5^{\circ}$ CONDITIONS D'EsSAI DES APPAREILS TESTÉs}

a) Poussoir MICRON « 5 (fig. 2)

La poudre de D.T.P.A. est dispersée dans du fréon grâce à de l'huile d'amandes douces. D'autres tensio-actifs (myristate d'isopropyle, labrafils et trioléate de Sorbitan) ont été essayés et ont donné de moins bons résultats quant à la suspension et à la distribution du D.T.P.A.

Le mélange suivant :

D.T.P.A. $\mathrm{CaNa}_{3} \ldots \ldots \ldots \ldots \ldots \ldots \ldots \ldots \ldots \ldots \ldots \ldots \ldots$

Huile d'amandes douces .............. $150 \mathrm{mg}$

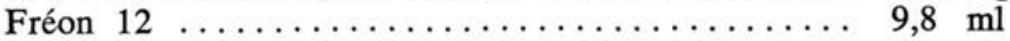

est conditionné en flacons de $12 \mathrm{ml}$ munis soit d'une valve doseuse de $100 \mathrm{~mm}^{3}$ (environ 100 bouffées par flacon), soit d'une valve continue. Le poussoir proprement dit est télescopique et à jet tourbillonnaire.

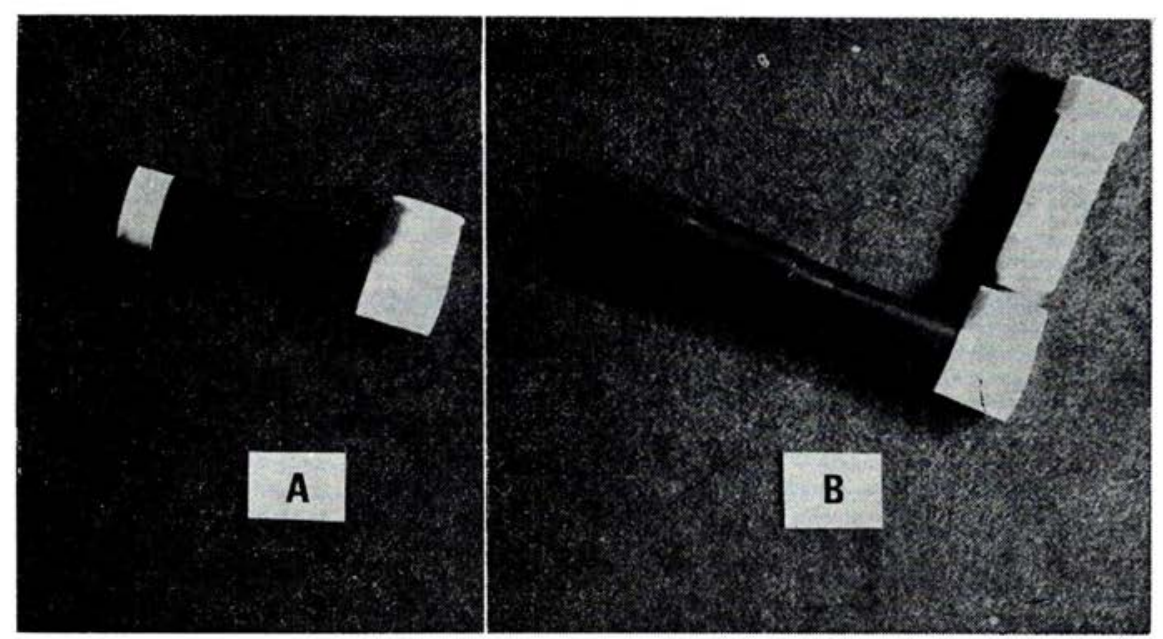

FIG. 2. - Poussoir MICRON \& 5 ,

a) Fermé

b) En ordre de marche.

vOL. $9-\mathrm{N}^{\circ} 3$ 
Le temps de maintien de la poudre en suspension est très bref et oblige à agiter le flacon entre chaque bouffée. Les valves sont prévues pour un fonctionnement inversé (corps percé) : le poussoir doit donc être actionné et relâché en position retournée (fig. $2 b$ ).

Pour les essais, le poussoir MICRON « 5 » a été placé en série dans le circuit inspiratoire du respirateur BIRD Mark 8 assurant le contrôle ventilatoire de l'animal intubé. Nous avons utilisé les appareils équipés d'une valve doseuse et délivré lors du temps inspiratoire soit 10 , soit 20 bouffées calibrées, une demi-heure après dépôt profond d'environ $10 \mu \mathrm{g}$ de lanthane sous forme de chlorure en solution chlorhydrique $0,1 \mathrm{~N}$. La méthode employée est la même que celle que nous avions préalablement appliquée à l'administration du D.T.P.A. en aérosol liquide [12].

b) Turbo-inhalateur SPINHALER (fig. 3)

La poudre de D.T.P.A. est conditionnée en capsules transparentes à couvercle coloré, à raison de $50 \mathrm{mg} /$ capsule.

Le turbo-inhalateur est composé d'un tube à étranglement dans lequel une petite hélice tourne et vibre à haute vitesse lors du passage de l'air inspiré. La capsule mise en place est solidaire de l'hélice. Un système coulissant en assure la perforation par deux poinçons, après un va-et-vient.
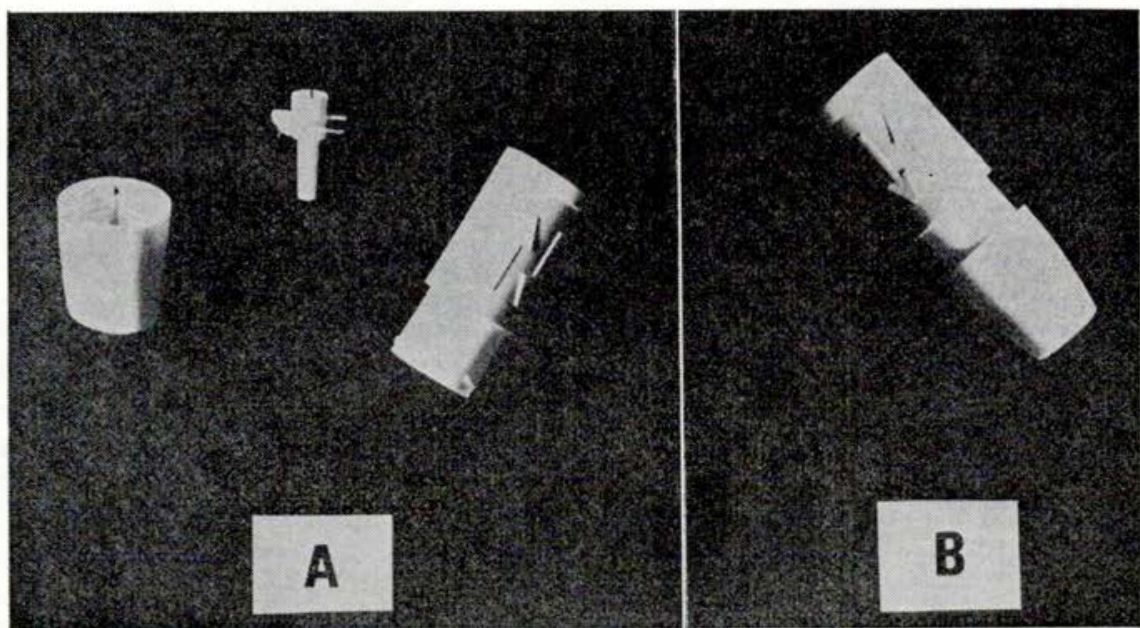

FIG. 3. - Turbo-inhalateur SPINHALER

a) Pièces détachées

b) En ordre de marche.

Les trous ainsi réalisés sont situés du côté de l'étranglement opposé à celui où se trouve l'hélice. Lors de l'inspiration, les vibrations de l'hélice provoquent la fluidisation de la poudre alors que sa rotation à grande vitesse crée une dépression (effet Venturi) qui aspire la poudre hors de la capsule. Les particules ainsi dispersées sont entraînées par l'air inspiré. 
Le problème posé était de vérifier que les particules de D.T.P.A. parvenaient dans une proportion intéressante jusqu'à l'alvéole pulmonaire. Le moyen de le résoudre était de contaminer un poumon avec du lanthane, d'administrer le D.T.P.A. au moyen du SPINHALER, $30 \mathrm{mn}$ après et de constater ou non une absorption du chélate D.T.P.A.-lanthane.

Pour cela, nous avons interposé le SPINHALER dans le circuit inspiratoire du respirateur BIRD Mark 8 comme nous l'avions fait précédemment pour le poussoir MICRON « 5 ». L'échec que nous avons subi s'explique par le mode de fonctionnement en relaxateur de pression du respirateur: celui-ci compense la dépression créée par l'hélice du SPINHALER et la poudre ne sort pas de la capsule. Devant l'impossibilité d'essayer l'appareil sur l'animal nous avons décidé de l'essayer sur un volontaire humain.

Le mode d'emploi du SPINHALER est alors le suivant (fig. 4) :
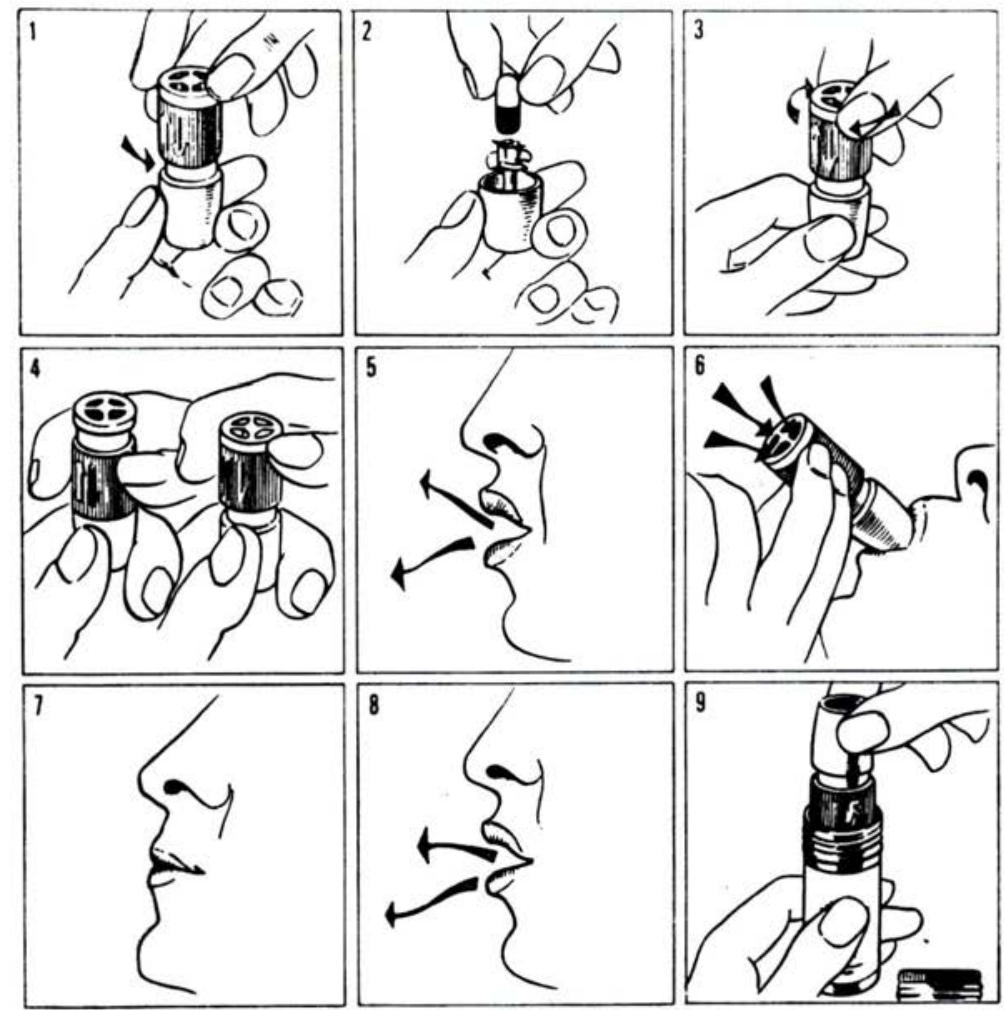

FIG. 4. - Mode d'emploi du SPINHALER

1. Tenir le SPINHaler en position verticale, l'embout buccal blanc vers le bas, et dévisser le corps tubulaire. 
2. Insérer l'extrémité colorée de la capsule dans la cupule de l'hélice ; s'assurer que l'hélice tourne librement.

3. Revisser le corps tubulaire.

4. Perforer la capsule en abaissant à fond le manchon gris muni de flèches et ramener celui-ci à sa position de départ.

5. Tenir 1'appareil loin de la bouche et vider les poumons au maximum.

6. Introduire l'embout buccal entre les dents en rejetant la tête en arrière ; serrer les lèvres ; inspirer profondément aussi rapidement que possible. On doit entendre la vibration de l'hélice.

7. Retenir le souffle pendant quelques secondes, puis retirer l'appareil de la bouche. capsule.

8. Répéter trois à quatre fois les opérations 5,6 et 7 pour vider la

9. Retirer la capsule vide et remettre l'appareil dans son emballage étanche. Il ne peut fonctionner de façon satisfaisante que s'il est propre et $s e c$. Le nettoyage se fait soit au pinceau en chassant la poudre déposée, soit par immersion dans l'eau chaude de l'appareil démonté. Il faut alors prendre soin de le sécher parfaitement.

\section{$6^{\circ}$ Mesure de L'efficacité du D.T.P.A.}

Pour une charge pulmonaire initiale de $10 \mu \mathrm{g}$, chez le singe de 3 à $5 \mathrm{~kg}$, le lanthane ne franchit la barrière alvéolo-capillaire que dans la proportion de 3 à $8 \%$ en $4 \mathrm{~h}$ [10]. Pour une même charge pulmonaire initiale, le D.T.P.A. administré en aérosol liquide et en large excès, $30 \mathrm{mn}$ après la contamination, provoque en $6 \mathrm{~h}$ l'absorption de 67 à $73 \%$ du dépôt pulmonaire profond [4]. Sur ces bases, nous pouvons apprécier l'efficacité des appareils testés en rapportant la fraction de lanthane absorbée en un temps donné à la quantité de lanthane présente dans le poumon au moment de l'administration du D.T.P.A. (fig. 5). Ce procédé de mesure de l'efficacité du D.T.P.A. sur les contaminations pulmonaires a été exposé et discuté par ailleurs [12].

\section{RESULTATS}

\section{$1^{\circ}$ POUSSOIR MICRON « 5 »}

A chaque manipulation correspond l'enregistrement de la radioactivité pulmonaire une demi-heure avant et $6 \mathrm{~h}$ après l'administration du D.T.P.A. comme le montre la figure 5. L'efficacité du traitement est exprimée par le pourcentage de la charge pulmonaire initiale corrigée du bruit de fond et de la décroissance physique du lanthane, quittant le poumon en $6 \mathrm{~h}$. Le flacon contenant $100 \mathrm{mg}$ de D.T.P.A. et étant vidé par environ 100 bouffées calibrées, chaque bouffée contient donc approximativement $1 \mathrm{mg}$ de D.T.P.A. 


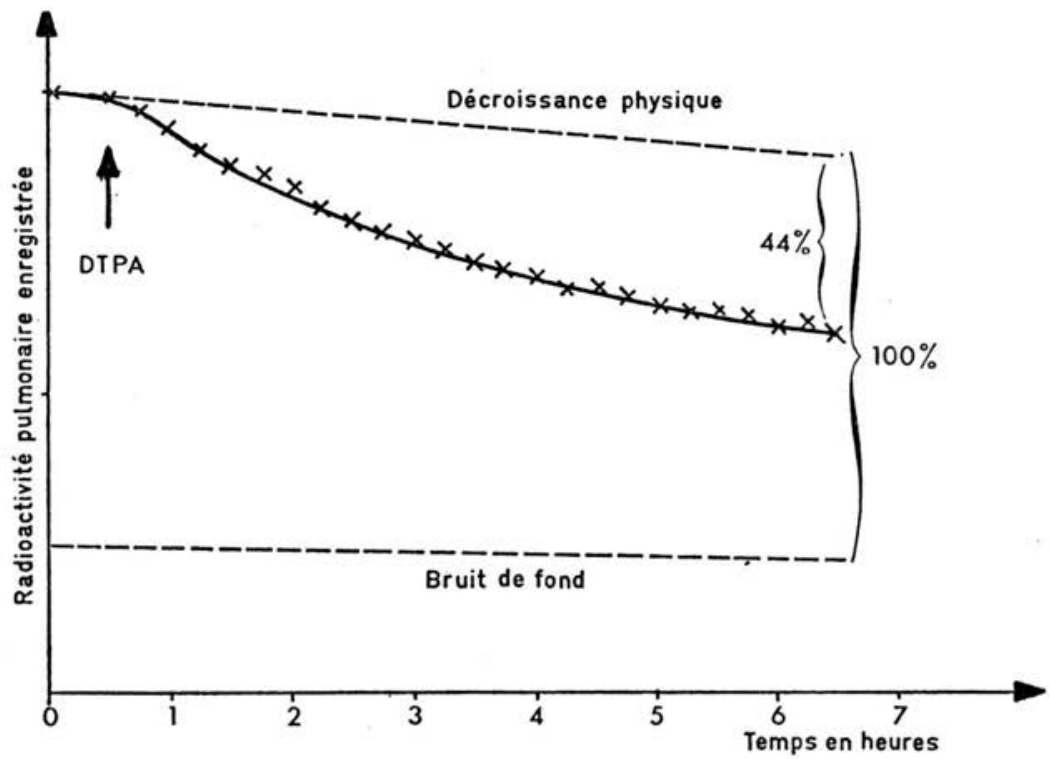

FIg. 5. - Effet du D.T.P.A. délivré par le poussoir MICroN \& 5 * sur la rétention pulmonaire du lanthane. Pour 10 bouffées de D.T.P.A. $44 \%$ de la charge pulmonaire initiale est éliminée en 6 heures (valeur corrigée du bruit de fond et de la décroissance physique du lanthane 140)

Nous avons obtenu les résultats regroupés dans le tableau I. Leur grande dispersion tient au fait que, la détente du fréon refroidissant l'ensemble du dispositif, l'huile d'amande douce se fige et colmate l'injecteur plus ou moins rapidement. On peut donc seulement dire que les aérosols délivrés par l'appareil parviennent effectivement jusqu'à l'alvéole pulmonaire, mais aucun calcul de rendement du dépôt alvéolaire ne peut être effectué, du fait des aléas de fonctionnement. La masse de D.T.P.A. en mg déposée par bouffée lors des expérimentations rapportées dans le tableau I et

TABLEAU I

ESSAIS DU POUSSOIR MICRON « 5 »: EFFICACITÉ DU TRAITEMENT

\begin{tabular}{|c|c|c|c|}
\hline \multicolumn{2}{|c|}{ Traitement } & 10 Bouffées & 20 Bouffées \\
\hline \multirow{5}{*}{ Efficacité } & $11-04-74^{*}$ & & \multirow[t]{2}{*}{$37,5(105)^{* *}$} \\
\hline & $12-04-74$ & 44 (99) & \\
\hline & $17-04-74$ & $\sim 0$ (105) & \multirow[b]{2}{*}{5,4 (99) } \\
\hline & $19-04-74$ & & \\
\hline & $\begin{array}{l}10-05-74 \\
13-05-74\end{array}$ & $9,2(105)$ & $26 \quad(99)$ \\
\hline
\end{tabular}

* Date de la manipulation.

** Numéro de l'animal.

voL. $9-\mathrm{N}^{\circ} 3$ 
calculée à l'aide de données acquises précédemment [12] serait de $0,3 \mu \mathrm{g}$ avec les valeurs extrêmes égales à 0 et 1 .

\section{$2^{\circ}$ TURBo-INHALATEUR SPINHALER}

Le résultat de l'expérimentation sur volontaire humain est donné par la figure 6 . Environ $50 \%$ de la charge pulmonaire initiale a quitté le poumon en $100 \mathrm{mn}$. Parallèlement, on observe un accroissement de la radioactivité vésicale. Il s'agit donc bien d'une absorption de lanthane chélaté par le D.T.P.A., ce qui implique pour celui-ci un dépôt alvéolaire notable. Le rendement de ce dépôt par rapport à la totalité du D.T.P.A. inhalé ne peut être déduit de cette expérimentation. En effet, les données qui nous permettraient de le faire concernent le singe Maccacus et non pas l'Homme [12]. Toutefois, pour un volume courant respiratoire de $1500 \mathrm{ml}$ et des particules de $2 \mu \mathrm{m}$ de diamètre, le dépôt pulmonaire profond est de $45 \%$ des particules inhalées [13]. Nous ne connaissons pas la valeur du volume courant respiratoire lors de notre essai du turbo-inhalateur, mais nous pouvons dire qu'elle était au moins égale à $1500 \mathrm{ml}$. Dans ces conditions, on peut considérer, compte tenu de la distribution de fréquence en dimensions des particules de D.T.P.A. (fig. 1) et des données de Hatch et Gross [13], que 10 à $20 \mathrm{mg}$ de D.T.P.A. ont atteint le compartiment pulmonaire profond à la suite de l'inhalation d'une capsule de $50 \mathrm{mg}$.

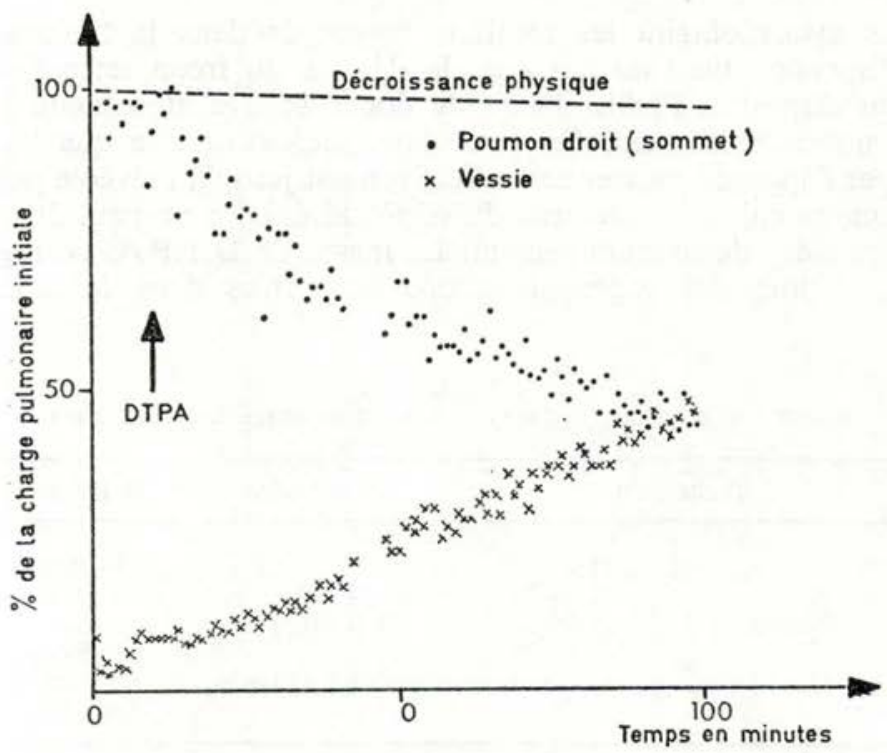

FIG. 6. - Effet du D.T.P.A. délivré par le turbo-inhalateur SPINHALER sur la rétention pulmonaire du lanthane et sur l'élimination urinaire 


\section{DISCUSSION}

La discussion va porter sur la réponse des deux appareils aux critères spécifiés en début d'exposé. Elle est résumée dans le tableau II.

\section{$1^{\circ}$ InNocuité DES ADJUVANTS}

L'utilisation du SPINHALER ne nécessite aucun adjuvant. Par contre, le MICRON « 5 » utilise le D.T.P.A. en suspension dans le fréon 12 additionné d'huile d'amandes douces. La toxicité du mélange à parties égales des fréons 11 et 12 a été étudiée par PAULET et coll. [14] et la parfaite innocuité du fréon 12 a été démontrée par les mêmes auteurs dans d'autres travaux non publiés. Par contre, il est peu souhaitable d'inhaler des composés huileux du fait de leur action inhibitrice des mouvement ciliaires de l'épithélium bronchique et du risque de formation de huilomes [15].

\section{TABLEAU II}

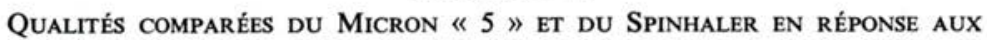
CRITÈRES D'UTILISATION

\begin{tabular}{|c|c|c|}
\hline Critères d'utilisation & MICRON « $5 »$ & SPINHALER \\
\hline Adjuvants & $\begin{array}{c}\text { Fréon } 12 \\
\text { Huile d'amandes } \\
\text { douces }\end{array}$ & \\
\hline Efficacité & + & +++ \\
\hline Taille & +++ & +++ \\
\hline Simplicité d'emploi & ++ & ++ \\
\hline Sûreté & 0 & ++ \\
\hline Conservation & \pm & ++ \\
\hline Prix de revient & $\begin{array}{l}\text { Environ } 2,00 \mathrm{~F} \text { pièce } \\
+ \text { fréon et DTPA } \\
\text { micronisé } \\
+ \text { conditionnement }\end{array}$ & $\begin{array}{l}\text { Environ } 10,00 \mathrm{~F} \text { pièce } \\
+ \text { conditionnement } \\
\text { en capsules du } \\
\text { DTPA micronisé. }\end{array}$ \\
\hline
\end{tabular}

\section{$2^{\circ}$ Efficacité}

A nombre d'inhalations égal, l'utilisation du SPINHALER conduit à un dépôt pulmonaire profond environ 1000 fois supérieur à celui obtenu avec le MICRON « 5 ».

\section{$3^{\circ}$ Taille}

Compte tenu du fait que le sPINHALER doit être accompagné d'un flacon contenant les capsules de poudre, les tailles sont comparables et répondent parfaitement à l'appellation « de poche » : 


$$
\begin{aligned}
& \text { MICRON \& } 5 \text { » }=2,5 \times 10 \mathrm{~cm} \\
& \text { SPINHALER }=2,5 \times 7 \mathrm{~cm}
\end{aligned}
$$

\section{$4^{\circ}$ Simplicité D'EMPLOI}

Le MICRON « 5》 doit être déployé, le flacon mis en place et agité avant chaque inhalation. Le SPINHALER doit être démonté (un quart de tour de pas de vis), la capsule, mise en place et l'appareil, remonté. La technique d'inhalation (tête rejetée en arrière et inspiration forcée) est la même pour les deux appareils. Ces opérations sont relativement simples pour chacun des deux dispositifs.

\section{$5^{\circ}$ SURETÉ DE FONCTIONNEMENT}

Le MICRON « 5 » fonctionne très convenablement lorsqu'on sollicite 1 ou 2 bouffées seulement. Lors d'un usage plus intensif, il se bloque après un nombre variable de bouffées, situé aux environs de 10.

Or, l'appareil est destiné au traitement d'urgence des contaminations respinatoires. Il doit être utilisé sur simple présomption de contamination, donc sans que l'on connaisse la charge pulmonaire. Dans ces conditions, aucune posologie ne pouvant être déterminée et compte tenu du faible rendement du dépôt pulmonaire profond avec cet appareil, il paraîtrait souhaitable de vider un flacon dans le délai le plus bref, ce qui est impossible.

Le SPINHALER par lui-même ne peut pas ne pas fonctionner. Le seul risque est celui d'une agglomération de la poudre dans les capsules. Ce problème semble avoir été résolu pour le médicament administré à l'aide du SPINHALER dans l'asthme allergique.

\section{$6^{\circ}$ CONSERVATION DU PRINCIPE ACTIF}

Dans les flacons du MICRON « 5 », nous avons constaté la formation de précipités collés aux parois et non redispersables dans la proportion de 4 flacons sur 20.

Le SPINHALER exige que l'appareil lui-même ainsi que les capsules soient conservés dans des conditions de sécheresse absolue afin d'éviter l'agglomération des particules entre elles ou de celles-ci dans l'appareil lors de l'inhalation.

\section{$7^{\circ}$ PRIX DE REVIENT}

Les deux appareils nécessitent du D.T.P.A. $\mathrm{CaNa}_{3}$ en poudre micronisée. Le SPINHALER par lui-même coûte environ 5 fois plus cher que le MICRON « 5»; mais le conditionnement en capsule est sûrement moins onéreux que la mise en suspension dans le fréon; ceci doit compenser cela et les deux dispositifs répondent également à ce dernier critère. 


\section{CONCLUSION}

L'absence d'adjuvants, la sûreté de fonctionnement et surtout la grande efficacité plaident en faveur du SPINHALER comme dispositif d'inhalation, individuel et portatif, susceptible d'être utilisé pour administrer un aérosol de D.T.P.A. en traitement d'urgence des contaminations respiratoires par les radionucléides relevant de cette thérapeutique.

Par le fait que l'on ne connaît pas la charge pulmonaire lors d'un traitement d'urgence, aucune posologie ne peut être fixée à l'avance. Toutefois, le calcul théorique nous a montré que, pour une poudre ayant des caractéristiques granulométriques identiques à la nôtre, 10 à $20 \mathrm{mg}$ de D.T.P.A. étaient susceptibles d'atteindre le poumon profond. L'inhalation du contenu de 5 capsules à la suite d'un incident nous paraît raisonnable.

\section{REMERCIEMENTS}

Nous tenons à remercier ici pour leur participation à ce travail, M. Kientzel (L.C.A., 94-Arcueil), M. Lefort des Ylouses (Faculté de Pharmacie, Chatenay-Malabry), Mme Roy et MM. Gongora (Fondation Curie, Paris).

\section{BIBLIOGRAPHIE}

[1] Catsch A. Radioactive metal mobilization in medecine. Ch. C. Thomas, Springfield, 1964.

[2] Morin M., Nenot J.C., Lafuma J. The behavior of ${ }^{237} \mathrm{~Np}$ in the rat. Health Phys., $1973,24,311-315$.

[3] Lafuma J. Commissariat à l'Energie Atomique, Département de Protection. Communication personnelle.

[4] Ducousso R., de Regnaud M., Perrault G., Pasquier C. DTPA therapy in the monkey of lung internal exposures to lanthanum : effect of therapeutic delays and routes of administration on treatment effectiveness. Dans : Health Physics problems of internal contamination, E. Bujdoso, Ed., Akademiai Kiado, Budapest, 1973, 321-325.

[5] Ducousso R., Nenot J.C., PASQuier C. Thérapeutique des radiocontaminations internes. Aspect actuel et perspectives. Radioprotection, 1974, 9, 27-29.

[6] Tombropoulos E.G., BaIR W.J. Treatment for removal of inhaled radioactive ceria $\left({ }^{144} \mathrm{CeO}_{2}\right)$ from the lungs of rats. Nature, 1962, 196, 82-83.

[7] LyUBChaNSKII E.R. Using $\mathrm{Na}_{3} \mathrm{Ca}$ DTPA (pentacin) to eliminate ${ }^{239} \mathrm{Pu}$ from the organism of the rat with inhalation poisoning. Dans : Distribution and biological effects of radioactive isotopes MOSKALEV Yu.I., Atomizdat, Moscou, 1966, 471-476. AEC-Tr-6944, 1968, 592-598.

[8] Ducousso R., Brazier J., Kress M., Pasquier C. DTPA et membrane alvéolo-capillaire : mécanismes du transport et de sa régulation. Path. Biol., à paraitre.

[9] Ducousso R., Bereziat G., Perrault G., Pasquier C. Influence de la charge pulmonaire en lanthane sur l'épuration précoce provoquée par un aérosol de DTPA. Health Phys., 1971, 21, 21-29.

[10] Ducousso R., PASquier C. Lung contamination by Ce and La. Evolution of the early spontaneous absorption as a function of the initial lung burden. Health Physics, 1974, 26, 519-524.

vol. $9-\mathrm{N}^{\circ} 3$ 
[11] Gongora G., Roy M., Gongota R., Jammet J., Magdelenat H., Jammet H., Drutel P., Castillon du Perron M., Verdier F., Parrot R., Boscus M. Méthode utilisée pour l'étude de la répartition de l'épuration pulmonaire chez l'homme normal, après administration d'aérosols radioactifs. J. Biol. Méd. Nucl., 1973, 8, 19-26.

[12] Ducousso R. Etudes des mécanismes de la mobilisation précoce des éléments non transférables au niveau du poumon après inhalation. Rapport CEA-R-4592. 1974 ,

[13] Hatch T.F., Gross P. Pulmonary deposition and retention of inhaled aerosols. Academic Press, New York, 1964.

[14] Paulet G., Desbrousses S., Sorais J. Toxicité chronique à moyen terme de deux hydrocarbures chlorofluorés : R 11 et R 12. Arch. Mal. Prof., 1967, 28, 464-469.

[15] Chrétien J. Faculté de Médecine de Créteil (94). Communication personnelle. 